\title{
TOPOLOGICAL TRIVIALITY OF FAMILIES OF FUNCTIONS ON ANALYTIC VARIETIES
}

\author{
MARIA APARECIDA SOARES RUAS AND \\ JOÃO NIVALDO TOMAZELLA
}

\begin{abstract}
We present in this paper sufficient conditions for the topological triviality of families of germs of functions defined on an analytic variety $V$. The main result is an infinitesimal criterion based on a convenient weighted inequality, similar to that introduced by T. Fukui and L. Paunescu in [8]. When $V$ is a weighted homogeneous variety, we obtain as a corollary, the topological triviality of deformations by terms of non negative weights of a weighted homogeneous germ consistent with $V$. Application of the results to deformations of Newton non-degenerate germs with respect to a given variety is also given.
\end{abstract}

\section{$\S 1$. Introduction}

Let $V, 0$ be the germ of an analytic subvariety of $k^{n}, k=\mathbb{R}$, or $\mathbb{C}$ and let $\mathcal{R}_{V}$ (respectively $C^{0}-\mathcal{R}_{V}$ ) be the group of germs of diffeomorphisms (respectively homeomorphisms) preserving $V, 0$, acting on germs $h_{0}: k^{n}, 0 \rightarrow k, 0$. The aim of this paper is to study topologically trivial deformations of $\mathcal{R}_{V^{-}}$ finitely determined germs $h_{0}$. The main result is Theorem 3.4 in which we introduce a sufficient condition for the $C^{0}-\mathcal{R}_{V}$-triviality of families of map germs $h: k^{n} \times k, 0 \rightarrow k, 0, h(x, 0)=h_{0}(x)$, based on a convenient weighted inequality, similar to that introduced by T. Fukui and L. Paunescu in [8]. A non weighted version of this result first appeared in [13]. There, the sufficient condition for topological triviality is formulated in terms of the integral closure of the tangent space to the $\mathcal{R}_{V}$-orbit of $h_{t}$.

As an application of the results, when $V$ is a weighted homogeneous analytic variety, we prove that any deformation by non negative weights of an $\mathcal{R}_{V}$-finitely determined weighted homogeneous germ (consistent with $V$ ) is topologically trivial. This result was previously proved by J. Damon

Received October 22, 2001.

2000 Mathematics Subject Classification: 32S15, 58K40, 58K15.

The first author was partially supported by Conselho Nacional de Desenvolvimento Científico e Tecnológico (CNPq), Brazil, grant \# 300066/88-0 and by FAPESP, grant \# 97/10735-3. 
in [6]. In the last section, we obtain sufficient conditions for the $C^{0}-\mathcal{R}_{V^{-}}$ triviality of families $h(x, t)=h_{0}(x)+t g(x)$, depending only on $h_{0}$. When $h_{0}$ is Newton non-degenerate with respect to the variety $V$ (see Definition 4.4), we describe the topological triviality of $h$ in terms of the Newton diagram of the tangent space to the $\mathcal{R}_{V}$-orbit of $h_{0}$.

For other results related to the subject discussed in this paper, see for instance [1], [6], [13].

\section{$\S 2$. Basic results}

Let $\mathcal{O}_{n}$ be the ring of germs of analytic functions $h: k^{n}, 0 \rightarrow k, k=\mathbb{R}$ or $\mathbb{C}$. This is a local ring with maximal ideal $\mathcal{M}_{n}$, the germs with zero target.

A germ of a subset $V, 0 \subset k^{n}, 0$ is the germ of an analytic variety if there exist germs of analytic functions $f_{1}, \ldots, f_{r}$ such that $V=\left\{x: f_{1}(x)=\cdots=\right.$ $\left.f_{r}(x)=0\right\}$.

Our aim is to study map germs $h: k^{n}, 0 \rightarrow k, 0$ under the equivalence relation that preserves the analytic variety $V, 0$. We say that two germs $h_{1}$ and $h_{2}: k^{n}, 0 \rightarrow k, 0$ are $\mathcal{R}_{V}$-equivalent (respectively $C^{0}-\mathcal{R}_{V}$-equivalent) if there exists germ of diffeomorphism (respectively homeomorphism) $\phi$ : $k^{n}, 0 \rightarrow k^{n}, 0$ with $\phi(V)=V$ and $h_{1} \circ \phi=h_{2}$. That is,

$$
\mathcal{R}_{V}=\{\phi \in \mathcal{R}: \phi(V)=V\}
$$

where $\mathcal{R}$ is the group of germs of diffeomorphisms of $k^{n}, 0$.

A one parameter deformation $h: k^{n} \times k, 0 \rightarrow k, 0$ of $h_{0}: k^{n}, 0 \rightarrow k, 0$ is topologically $\mathcal{R}_{V}$-trivial (or $C^{0}-\mathcal{R}_{V}$-trivial) if there exists homeomorphism $\varphi: k^{n} \times k, 0 \rightarrow k^{n} \times k, 0, \varphi(x, t)=(\bar{\varphi}(x, t), t)$, such that $h \circ \varphi(x, t)=h_{0}(x)$ and $\varphi(V \times k)=V \times k$.

We denote by $\theta_{n}$ the set of germs of tangent vector fields in $k^{n}, 0 ; \theta_{n}$ is a free $\mathcal{O}_{n}$ module of rank $n$. Let $I(V)$ be the ideal in $\mathcal{O}_{n}$ consisting of germs of analytic functions vanishing on $V$. We denote by $\Theta_{V}=\left\{\eta \in \theta_{n}\right.$ : $\eta(I(V)) \subseteq I(V)\}$, the submodule of germs of vector fields tangent to $V$ (see [1] for more details).

The tangent space to the action of the group $\mathcal{R}_{V}$ is $T \mathcal{R}_{V}(h)=d h\left(\Theta_{V}^{0}\right)$, where $\Theta_{V}^{0}$ is the submodule of $\Theta_{V}$ given by the vector fields that are zero at zero. When the point $x=0$ is a stratum in the logarithmic stratification of the analytic variety, this is the case when $V$ has an isolated singularity at the origin (see [1] for details), both spaces $\Theta_{V}$ and $\Theta_{V}^{0}$ coincide. 
The group $\mathcal{R}_{V}$ is a geometric subgroup of the contact group, as defined by J. Damon [3], [4], hence the infinitesimal criterion for $\mathcal{R}_{V}$-determinacy holds (see [1] for a proof).

TheOREM 2.1. ([1]) The germ $h: k^{n}, 0 \rightarrow k, 0$ is $\mathcal{R}_{V}$-finitely determined if and only if there exists a positive integer $k$ such that $T \mathcal{R}_{V}(h) \supset$ $\mathcal{M}_{n}^{k}$

The following theorem is the geometric criterion for the $\mathcal{R}_{V}$-finite determinacy.

THEOREM 2.2. ([1]) Let $V, 0 \subseteq \mathbb{C}^{n}, 0$ be the germ of an analytic variety and let $h: \mathbb{C}^{n}, 0 \rightarrow \mathbb{C}, 0$ be the germ of an analytic function. Let

$$
V(h)=\left\{x \in \mathbb{C}^{n}: \xi h(x)=0, \forall \xi \in \Theta_{V}\right\} .
$$

Then $h$ is $\mathcal{R}_{V}$-finitely determined if and only if $V(h)=\{0\}$ or $\emptyset$.

As a consequence of this result, it follows that if $h$ is $\mathcal{R}_{V}$-finitely determined, then $h^{-1}(c)$ is transverse to $V$ away from 0 , for sufficiently small values of $c$.

In the real case, the necessary condition remains true, that is, if $h$ is $\mathcal{R}_{V}$-finitely determined then the set $\left\{x \in \mathbb{R}^{n}: \xi h(x)=0, \forall \xi \in \Theta_{V}\right\}$ is $\{0\}$ or $\emptyset$.

\section{$\S 3$. The main result}

Let $h_{0}: k^{n}, 0 \rightarrow k, 0$ be a $\mathcal{R}_{V}$-finitely determined germ of analytic function and let $h: k^{n} \times k, 0 \rightarrow k, 0$ be an analytic deformation of $h_{0}$. In the sequel, we shall assume $h(0, t)=0$. The property of being $\mathcal{R}_{V}$-finitely determined is open in the sense that the germ $\left\{x \in k^{n}: d h_{t} \xi(x)=0, \forall \xi \in\right.$ $\left.\Theta_{V}\right\}$ at 0 is $\{0\}$ or empty for sufficiently small values of the parameters (see [1]). However, this does not guarantee the existence of a neighbourhood $U$ of 0 in $k^{n}, 0$ and an open $\varepsilon$-ball, $B_{\varepsilon}$, centered at the origin in $k$ such that the above condition holds $\forall x \in U$ and $\forall t \in B_{\varepsilon}$. We then need the following definition:

Definition 3.1. Let $h_{0}: k^{n}, 0 \rightarrow k, 0$ be a $\mathcal{R}_{V}$-finitely determined germ. We say that a deformation $h: k^{n} \times k, 0 \rightarrow k, 0$ of $h_{0}$ is a good deformation if $V(h) \subseteq\{0\} \times k, 0$, where $V(h)=\left\{(x, t) \in k^{n} \times k, 0: d h_{t}(x) \xi(x)=\right.$ $\left.0, \forall \xi \in \Theta_{V}\right\}$. 
ExAMPLE 3.2. Let $V$ be the $x$-axis in $k^{2} ; \Theta_{V}$ is generated by $(1,0)$

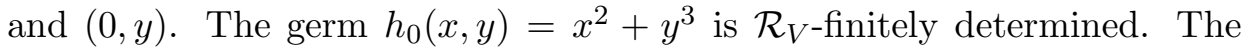
deformation $h_{t}(x, y)=x^{2}+y^{3}+t y^{2}$ of $h_{0}$ has the property that $h_{t}$ is $\mathcal{R}_{V^{-}}$ finitely determined for each fixed $t$, but we cannot find $\varepsilon>0$ such that the above condition holds for all $t \in B_{\varepsilon}$.

In what follows we can assume that $d h_{t} \xi(0)=0, \forall \xi \in \Theta_{V}$. In fact, if $\xi \in \Theta_{V}$, then $d h_{t} \xi \cdot \frac{\partial h}{\partial t}=d h_{t}\left(\frac{\partial h}{\partial t} \cdot \xi\right)$. If $d h_{t} \xi_{0}(0) \neq 0$ for some $\xi_{0}$, then $\frac{\partial h}{\partial t}=$ $d h_{t}\left(\frac{\partial h}{\partial t} \cdot \xi_{0}\right)$ and hence the deformation is $C^{\omega} \xi_{-} \mathcal{R}_{V}$-trivial (i.e. analytically trivial). Observe that $\frac{\frac{\partial h}{\partial t} \cdot \xi_{0}}{d h_{t} \xi_{0}} \in \Theta_{V}^{0}$.

Definition 3.3. (a) We assign weights $w_{1}, \ldots, w_{n}, w_{i} \in \mathbb{Z}^{+}, i=$ $1, \ldots, n$ to a given coordinate system $x_{1}, \ldots, x_{n}$ in $k^{n}$. The filtration of a monomial $x^{\beta}=x_{1}^{\beta_{1}} x_{2}^{\beta_{2}} \cdots x_{n}^{\beta_{n}}$ with respect to this set of weights is defined by $\operatorname{fil}\left(x^{\beta}\right)=\sum_{i=1}^{n} \beta_{i} w_{i}$.

(b) We define a filtration in the $\operatorname{ring} \mathcal{O}_{n}$ via the function

$$
\operatorname{fil}(f)=\inf _{|\beta|}\left\{\operatorname{fil}\left(x^{\beta}\right): \frac{\partial^{|\beta|} f}{\partial x^{\beta}}(0) \neq 0\right\}, \quad|\beta|=\beta_{1}+\cdots+\beta_{n} .
$$

The filtration of a map germ $f=\left(f_{1}, \ldots, f_{p}\right): k^{n}, 0 \rightarrow k^{p}, 0$ is $\operatorname{fil}(f)=$ $\left(d_{1}, \ldots, d_{p}\right)$, where $\operatorname{fil}\left(f_{i}\right)=d_{i}$.

(c) We extend the filtration to $\Theta_{V}$, defining $w\left(\frac{\partial}{\partial x_{j}}\right)=-w_{j}$ for all $j=$ $1, \ldots, n$, so that given $\xi=\sum_{j=1}^{n} \xi_{j} \frac{\partial}{\partial x_{j}} \in \Theta_{V}$, then $\operatorname{fil}(\xi)=\inf _{j}\left\{\operatorname{fil}\left(\xi_{j}\right)-w_{j}\right\}$.

(d) Given $\left(w_{1}, \ldots, w_{n}: d_{1}, \ldots, d_{p}\right), w_{i}, d_{j} \in \mathbb{Z}^{+}$, a map germ $f$ : $k^{n}, 0 \rightarrow k^{p}, 0$ is weighted homogeneous of type $\left(w_{1}, \ldots, w_{n}: d_{1}, \ldots, d_{p}\right)$ if for all $\lambda \in k-\{0\}$ :

$$
f\left(\lambda^{w_{1}} x_{1}, \lambda^{w_{2}} x_{2}, \ldots, \lambda^{w_{n}} x_{n}\right)=\left(\lambda^{d_{1}} f_{1}(x), \lambda^{d_{2}} f_{2}(x), \ldots, \lambda^{d_{p}} f_{p}(x)\right) .
$$

Let $w=w_{1} w_{2} \cdots w_{n}, \mathbf{w}=\left(w_{1}, \ldots, w_{n}\right)$, and $\|x\|_{\mathbf{w}}=\left(\left|x_{1}\right|^{2 w / w_{1}}+\cdots+\right.$ $\left.\left|x_{n}\right|^{2 w / w_{n}}\right)^{1 / 2 w}$.

In what follows $A \lesssim B$ means there is some positive constant $C$ with $A \leq C B$.

Our main result is the following theorem: 
THEOREM 3.4. Let $\mathbf{w}=\left(w_{1}, \ldots, w_{n}\right)$ be an $n$-tuple of positive integers. Let $\alpha_{1}, \ldots, \alpha_{m}$ be a system of generators for $\Theta_{V}^{0}$ and $d_{i}=\operatorname{fil}\left(\alpha_{i}\right), i=$ $1, \ldots, m$. Let $h_{0}: k^{n}, 0 \rightarrow k, 0$ be a $\mathcal{R}_{V}$-finitely determined germ and $h$ : $k^{n} \times k, 0 \rightarrow k, 0$ a good deformation of $h_{0}$. If

$$
\left|\frac{\partial h}{\partial t}\right| \lesssim \sup _{i=1, \ldots, m}\left\{\left|d h_{t}\left(\alpha_{i}\right)\right|\|x\|_{\mathbf{w}}^{-d_{i}}\right\} \quad \text { for } x(\neq 0) \text { near } 0
$$

then $h$ is $C^{0}-\mathcal{R}_{V}$-trivial.

Proof. We choose non negative integers $e_{i}, i=1, \ldots, m$ so that $d_{i}+e_{i}$ is a constant $s$. We define a function $\rho$ by $\rho^{2}=\sum_{i=1}^{m}\left|\rho_{i}\right|^{2}\|x\|_{\mathbf{w}}^{2 e_{i}}$, where $\rho_{i}=d h_{t}\left(\alpha_{i}\right), i=1, \ldots, m$. Since $h$ is a good deformation it follows that $V(\rho(x, t))=\{0\} \times k$. From the equation $\rho^{2} \frac{\partial h}{\partial t}=d h_{t}\left(\frac{\partial h}{\partial t} \sum_{i=1}^{m} \overline{\rho_{i}}\|x\|_{\mathbf{w}}^{2 e_{i}} \alpha_{i}\right)$, we obtain $d h(X)=0$, where $X$ is the vector field in $k^{n} \times k, 0$ defined by

$$
X(x, t)= \begin{cases}-\frac{1}{\rho^{2}} \frac{\partial h}{\partial t}\left(\overline{\rho_{1}}\|x\|_{\mathbf{w}}^{2 e_{1}} \alpha_{1}+\cdots+\overline{\rho_{m}}\|x\|_{\mathbf{w}}^{2 e_{m}} \alpha_{m}\right)+\frac{\partial}{\partial t} & \text { if } x \neq 0 \\ \frac{\partial}{\partial t} & \text { if } x=0\end{cases}
$$

The vector field $X(x, t)$ is real analytic away from $\{0\} \times k$. For $j=1, \ldots, n$ and $i=1, \ldots, m$, let $X_{j}$ denote the $j$-th component of $X$, and let $\alpha_{i j}$ denote the $j$-th component of $\alpha_{i}$. Then

$$
X_{j}(x, t)=-\frac{1}{\rho^{2}} \frac{\partial h}{\partial t}\left(\overline{\rho_{1}}\|x\|_{\mathbf{w}}^{2 e_{1}} \alpha_{1 j}+\cdots+\overline{\rho_{m}}\|x\|_{\mathbf{w}}^{2 e_{m}} \alpha_{m j}\right) .
$$

Since fil $\left(\alpha_{i}\right)=d_{i}$, we have $\operatorname{fil}\left(\alpha_{i j}\right) \geq d_{i}+w_{j}$, thus $\left|\alpha_{i j}\right| \lesssim\|x\|_{\mathbf{w}}^{d_{i}+w_{j}}$. Then,

$$
\begin{aligned}
\left|X_{j}(x, t)\right| & \lesssim \frac{1}{\rho}\left|\frac{\partial h}{\partial t}\right|\|x\|_{\mathbf{w}}^{e_{1}}\|x\|_{\mathbf{w}}^{d_{1}+w_{j}}+\cdots+\frac{1}{\rho}\left|\frac{\partial h}{\partial t}\right|\|x\|_{\mathbf{w}}^{e_{m}}\|x\|_{\mathbf{w}}^{d_{m}+w_{j}} \\
& \lesssim \frac{1}{\rho}\left|\frac{\partial h}{\partial t}\right|\|x\|_{\mathbf{w}}^{s}\|x\|_{\mathbf{w}}^{w_{j}} \lesssim \frac{1}{\rho} \sup _{i}\left\{\left|\rho_{i}\right|\|x\|_{\mathbf{w}}^{-d_{i}}\right\}\|x\|_{\mathbf{w}}^{s}\|x\|_{\mathbf{w}}^{w_{j}} \lesssim\|x\|_{\mathbf{w}}^{w_{j}}
\end{aligned}
$$

It follows that $\left|X_{j}(x, t)\right| \leq C\|x\|_{\mathbf{w}}^{w_{j}}$, for $j=1, \ldots, n$ and this implies that the vector field $X$ is integrable. In the real case a proof follows from $[8$, p. 87]. For completeness we include below a proof which holds both for the real and complex case. 
Lemma 3.5. Let

$$
X(x, t)= \begin{cases}\sum_{j=1}^{n} X_{j}(x, t) \frac{\partial}{\partial x_{j}}+\frac{\partial}{\partial t} & \text { if } x \neq 0 \\ \frac{\partial}{\partial t} & \text { if } x=0\end{cases}
$$

be a vector field in $k^{n} \times k, 0$, such that $X_{j}$ are real analytic away from $0 \times k$ and there exists $C>0$ with $\left|X_{j}(x, t)\right| \leq C\|x\|_{\mathbf{w}}^{w_{j}}$ for all $j=1, \ldots, n$. Then $X(x, t)$ is locally integrable in a neighbourhood of $(0,0) \in k^{n} \times k$.

Proof. The vector field $X$ is real analytic away from $0 \times k$. We only need to prove the uniqueness of the solutions at $(0, t)$. In fact, $\phi(\tau)=(0, \tau+t)$ is an integral curve of $X$ such that $\phi(0)=(0, t)$. Let $\varphi(\tau)=(x(\tau), t(\tau))$, be another integral curve with initial condition $\varphi(0)=(0, t)$. Since $x(0)=0$, $x_{j}(0)=0$, for all $j=1, \ldots, n$. Then

$$
x_{j}(\tau)=\int_{0}^{\tau} \frac{\partial \varphi_{j}}{\partial s} d s=\int_{0}^{\tau} X_{j}(x(s), t(s)) d s
$$

and

$$
\left|x_{j}(\tau)\right| \leq \int_{0}^{\tau}\left|X_{j}(x(s), t(s))\right| d s \leq \int_{0}^{\tau} C\|x(s)\|_{\mathbf{w}}^{w_{j}} d s .
$$

Therefore

$$
\begin{aligned}
\|x(\tau)\|_{\mathbf{w}}^{2 w} & =\sum_{j=1}^{n}\left|x_{j}(\tau)\right|^{2 w / w_{j}} \leq \sum_{j=1}^{n}\left(\int_{0}^{\tau}\|x(s)\|_{\mathbf{w}}^{w_{j}} d s\right)^{2 w / w_{j}} \\
& \leq n \int_{0}^{\tau}\|x(s)\|_{\mathbf{w}}^{2 w} d s .
\end{aligned}
$$

By the Gronwall's inequality, it follows that $x(\tau)=0$. Thus $\varphi(\tau)=$ $(0, t(\tau))$. However,

$$
\frac{d}{d \tau}(\phi(\tau)-\varphi(\tau))=X(0, \tau+t)-X(0, t(\tau))=0
$$

therefore $t(\tau)=\tau+t$ and $\varphi \equiv \phi$.

The following corollary of Theorem 3.4 follows when we consider the trivial filtration $w_{i}=1, i=1, \ldots, n$ in $k^{n}$. 
Corollary 3.6. Let $h_{0}: k^{n}, 0 \rightarrow k, 0$ be a $\mathcal{R}_{V}$-finitely determined germ and $h: k^{n} \times k, 0 \rightarrow k, 0$ a good deformation of $h_{0}$. If $\left|\frac{\partial h}{\partial t}\right| \lesssim$ $\sup _{i}\left\{\left|d h_{t}\left(\alpha_{i}\right)\right|\right\}$, then $h$ is $C^{0}-\mathcal{R}_{V}$-trivial.

This result first appeared in [13], but there the sufficient condition for topological triviality was formulated in terms of the integral closure of the ideal $\left\langle d h_{t}\left(\alpha_{i}\right)\right\rangle$.

Definition 3.7. A germ of an analytic variety $V, 0 \subseteq k^{n}, 0$ is weighted homogeneous if it is defined by a weighted homogeneous map germ $f$ : $k^{n}, 0 \rightarrow k^{p}, 0$. A set of generators $\left\{\alpha_{1}, \ldots, \alpha_{m}\right\}$ of $\Theta_{V}$ is weighted homogeneous of type $\left(w_{1}, \ldots, w_{n}: d_{1}, \ldots, d_{m}\right)$ if $\alpha_{i j}(i=1, \ldots, m, j=1, \ldots, n)$ are weighted homogeneous polynomials of type $\left(w_{1}, \ldots, w_{n}: d_{i}+w_{j}\right)$ whenever $\alpha_{i j} \neq 0$, where $\alpha_{i}=\sum_{j=1}^{n} \alpha_{i j} \frac{\partial}{\partial x_{j}}$.

When $V$ is a weighted homogeneous variety, we can always choose weighted homogeneous generators for $\Theta_{V}$ (see [7]).

Definition 3.8. ([5]) Let $V$ be defined by weighted homogeneous polynomials. We say that $h$ is weighted homogeneous consistent with $V$ if $h$ is weighted homogeneous with respect to the same set of weights assigned to $V$.

EXAMPLE 3.9. Let $V=\phi^{-1}(0) \subset k^{3}$ where $\phi(x, y, z)=2 x^{2} y^{2}+y^{3}-$ $z^{2}+x^{4} y$. We have $\phi$ is weighted homogeneous with respect to the weights $w_{1}=1, w_{2}=2, w_{3}=3$. Let $h(x, y, z)=x^{3}+x y+z$ and $f(x, y, z)=$ $x^{3}+x y+z^{2}$. Then $h$ is consistent with $V, f$ is weighted homogeneous but not consistent with $V$.

The following result was previously proved by J. Damon in [6]. We include it here as a corollary of Theorem 3.4.

Corollary 3.10. Let $V$ be a weighted homogeneous subvariety of $k^{n}, 0$ and let $h_{0}: k^{n}, 0 \rightarrow k, 0$ be weighted homogeneous consistent with $V$ and $\mathcal{R}_{V}$-finitely determined. Then any deformation $h$ of $h_{0}$ by terms of filtration greater than or equal to the filtration of $h_{0}$ is $C^{0}-\mathcal{R}_{V}$-trivial.

Proof. Let $\left\{\alpha_{1}, \ldots, \alpha_{m}\right\}$ be a set of weighted homogeneous generators of $\Theta_{V}$, and $d_{i}=\operatorname{fil}\left(\alpha_{i j}\right)-w_{j}$. Under the above conditions, $d h_{0}\left(\alpha_{i}\right)$ and $\rho^{2}(x, 0)=\sum_{i=1}^{m}\left|d h_{0}\left(\alpha_{i}\right)\right|^{2}\|x\|_{\mathbf{w}}^{2 e_{i}}$ are both weighted homogeneous. Since $h_{0}$ 
is $\mathcal{R}_{V}$-finitely determined, it follows that $\rho^{2}(x, 0)$ has isolated singularity at zero in $k^{n}$. Moreover, $\rho^{2}(x, t)$ is a deformation of $\rho^{2}(x, 0)$ by terms of filtration greater than or equal to the filtration $h_{0}$. Then there exist positive constants $c_{1}, c_{2}$ such that $c_{1} \rho^{2}(x, 0) \leq \rho^{2} \leq c_{2} \rho^{2}(x, 0)$ and thus $h$ is a good deformation of $h_{0}$ (see [11, Lemma 3]), for $t$ sufficiently close to zero.

Now fil $\left(\frac{\partial h}{\partial t}\right) \geq \operatorname{fil}\left(h_{0}\right)$ and

$$
\operatorname{fil}\left(d h_{t}\left(\alpha_{i}\right)\|x\|_{\mathbf{w}}^{-d_{i}}\right)=\operatorname{fil}\left(h_{0}\right)-w_{j}+\left(d_{i}+w_{j}\right)+\left(-d_{i}\right)=\operatorname{fil}\left(h_{0}\right) .
$$

Since $h$ is a good deformation of $h_{0}$, it follows that

$$
\left|\frac{\partial h}{\partial t}\right| \lesssim \sup _{i}\left\{\left|d h_{t}\left(\alpha_{i}\right)\right|\|x\|_{\mathbf{w}}^{-d_{i}}\right\},
$$

and result follows by Theorem 3.4.

ExAmPle 3.11. Let $V, 0 \subset \mathbb{R}^{3}, 0$ (or $\mathbb{C}^{3}, 0$ ) be defined by $\varphi(x, y, z)=$ $2 x^{k+1} y^{2}+y^{3}-z^{2}+x^{2(k+1)} y=0$. This is the implicit equation for the $S_{k^{-}}$ singularities classified by D. Mond [10]. The function germ $\varphi$ is weighted homogeneous of weights $2,2 k+2$ and $3 k+3$ for $x, y$ and $z$ respectively. We have that $h(x, y, z)=y+a_{k+1} x^{k+1}$ is $\mathcal{R}_{V}$-finitely determined for $a_{k+1} \neq 0,1$ and consistent with $V$. Therefore deformations of $h$ by terms of order higher than or equal to fil $(h)$ are $C^{0}-\mathcal{R}_{V}$-trivial. For $k$ odd, $h_{1}(x, y, z)=$ $z+a x^{3(k+1) / 2}$ and $h_{2}(x, y, z)=z+b x^{(k+1) / 2} y$ are consistent with $V$ and $\mathcal{R}_{V}$-finite for all $a^{2} \neq-4 / 27$ and $b \neq \pm 2$. Thus deformations of $h_{1}$ and $h_{2}$, respectively by terms of order higher than or equal to fil $\left(h_{1}\right)$ and $\operatorname{fil}\left(h_{2}\right)$ are $C^{0}-\mathcal{R}_{V}$-trivial.

The following example shows that the hypothesis in Corollary 3.10 can hold even when the condition $\left|\frac{\partial h}{\partial t}\right| \lesssim \sup _{i}\left\{\left|d h_{t}\left(\alpha_{i}\right)\right|\right\}$ does not hold.

ExAMPLE 3.12. Taking $k=1$ in the above example, the module $\Theta_{V}$ is generated by $\alpha_{1}=(2 x, 4 y, 6 z), \alpha_{2}=\left(0,2 z, x^{4}+4 x^{2} y+3 y^{2}\right), \alpha_{3}=$ $\left(x^{2}+3 y,-4 x y, 0\right)$ and $\alpha_{4}=\left(z, 0,2 x^{3} y+2 x y^{2}\right)$. Any deformation of the germ $h_{0}(x, y, z)=y+a x^{2}, a \neq 0,1$ by terms of filtrations higher than or equal to fil $\left(h_{0}\right)=2$ are $\mathcal{R}_{V}$-topologically trivial. In particular $h(x, y, z, t)=$ $y+(a+t) x^{2}$ is $\mathcal{R}_{V}$-topologically trivial. However the condition $\left|\frac{\partial h}{\partial t}\right|=$ $\left|x^{2}\right| \lesssim \sup _{i}\left\{\left|d h_{t}\left(\alpha_{i}\right)\right|\right\}$ does not hold. In fact, one can easily check that it fails along the curve $\phi: k, 0 \rightarrow k^{4}, 0, \phi(s)=\left(s,-a s^{2}, 0,0\right)$. 


\section{§4. Topological triviality and Newton polyhedron}

In this section, we study the $C^{0}-\mathcal{R}_{V}$-triviality of deformations $h(x, t)=$ $h_{0}(x)+\operatorname{tg}(x)$ of a $\mathcal{R}_{V}$-finitely determined germ $h_{0}$. Our sufficient conditions depend only on $h_{0}$, so they can be handled more easily than the hypothesis of Theorem 3.4.

The first result is the following theorem.

THEOREM 4.1. Let $\mathbf{w}=\left(w_{1}, \ldots, w_{n}\right)$ be an $n$-tuple of positive integers. Let $\alpha_{1}, \ldots, \alpha_{m}$ be a system of generators for $\Theta_{V}^{0}$ and $d_{i}=\operatorname{fil}\left(\alpha_{i}\right), i=$ $1, \ldots, m$. Let $h(x, t)=h_{0}(x)+\operatorname{tg}(x)$ be a deformation of a $\mathcal{R}_{V}$-finitely determined germ $h_{0}$ satisfying the following conditions:

(a) $|g| \lesssim \sup _{i}\left\{\left|d h_{0}\left(\alpha_{i}\right)\right|\|x\|_{\mathbf{w}}^{-d_{i}}\right\} ;$

(b) $\left|d g\left(\alpha_{j}\right)\right| \lesssim \sup _{i}\left\{\left|d h_{0}\left(\alpha_{i}\right)\right|\|x\|_{\mathbf{w}}^{-d_{i}}\right\}$ for $x(\neq 0)$ near 0 and all $j=$ $1, \ldots, m$.

Then $h$ is $C^{0}-\mathcal{R}_{V}$-trivial.

The proof of the theorem will follow from the Theorem 3.4 and the Lemma below.

LEMMA 4.2. Let $h$ be as above. If $\left|d g\left(\alpha_{j}\right)\right| \lesssim \sup _{i}\left\{\left|d h_{0}\left(\alpha_{i}\right)\right|\|x\|_{\mathbf{w}}^{-d_{i}}\right\}$ for $x(\neq 0)$ near 0 and all $j=1, \ldots, m$, then $h$ is a good deformation of $h_{0}$. Moreover, if $|g| \lesssim \sup _{i}\left\{\left|d h_{0}\left(\alpha_{i}\right)\right|\|x\|_{\mathbf{w}}^{-d_{i}}\right\}$ then $|g| \lesssim \sup _{i}\left\{\left|d h_{t}\left(\alpha_{i}\right)\right|\|x\|_{\mathbf{w}}^{-d_{i}}\right\}$.

Proof. By hypothesis there exist a neighbourhood $U$ of 0 in $k^{n}$ and a constant $C>0$ such that

$$
|t|\left|d g\left(\alpha_{j}\right)\right| \leq|t| C \sup _{i}\left\{\left|d h_{0}\left(\alpha_{i}\right)\right|\|x\|_{\mathbf{w}}^{-d_{i}}\right\} .
$$

On the other hand,

$$
\begin{aligned}
\sup _{i}\left\{\left|d h_{t}\left(\alpha_{i}\right)\right|\|x\|_{\mathbf{w}}^{-d_{i}}\right\} & =\sup _{i}\left\{\left|d h_{0}\left(\alpha_{i}\right)+t d g\left(\alpha_{i}\right)\right|\|x\|_{\mathbf{w}}^{-d_{i}}\right\} \\
& \geq \sup _{i}\left\{\left|d h_{0}\left(\alpha_{i}\right)\right|\|x\|_{\mathbf{w}}^{-d_{i}}\right\}-|t| \sup _{i}\left\{\left|d g\left(\alpha_{i}\right)\right|\|x\|_{\mathbf{w}}^{-d_{i}}\right\} \\
& \geq \sup _{i}\left\{\left|d h_{0}\left(\alpha_{i}\right)\right|\|x\|_{\mathbf{w}}^{-d_{i}}\right\}-|t| C \sup _{i}\left\{\left|d h_{0}\left(\alpha_{i}\right)\right|\|x\|_{\mathbf{w}}^{-d_{i}}\right\} \\
& \geq(1-\beta) \sup _{i}\left\{\left|d h_{0}\left(\alpha_{i}\right)\right|\|x\|_{\mathbf{w}}^{-d_{i}}\right\}
\end{aligned}
$$

for some $0<\beta<1$ and $|t| \leq \beta / C$. Thus,

$$
\sup _{i}\left\{\left|d h_{t}\left(\alpha_{i}\right)\right|\|x\|_{\mathbf{w}}^{-d_{i}}\right\} \geq K \sup _{i}\left\{\left|d h_{0}\left(\alpha_{i}\right)\right|\|x\|_{\mathbf{w}}^{-d_{i}}\right\}
$$

for $t$ sufficiently small and $K>0$ and this implies the result. 
Before stating the next result, we recall the basic notions of Newton polyhedron of an ideal.

The Newton polyhedron of an ideal in $\mathcal{O}_{n}$ is defined as follows (see [9], [12]). We fix a coordinate system $x$ in $k^{n}$, so that $\mathcal{O}_{n}$ is identified with the ring $k\{x\}$ of convergent power series. For each germ $g(x)=\sum a_{r} x^{r}$, we define $\operatorname{supp} g=\left\{r \in \mathbb{Z}^{n}: a_{r} \neq 0\right\}$.

Definition 4.3. (i) Let $I$ be an ideal in $\mathcal{O}_{n}$, define

$$
\operatorname{supp} I=\bigcup\{\operatorname{supp} g: g \in I\} \text {. }
$$

(ii) The Newton polyhedron of $I$, denoted by $\Gamma_{+}(I)$, is the convex hull in $\mathbb{R}_{+}^{n}$ of the set

$$
\bigcup\left\{r+v: r \in \operatorname{supp} I, v \in \mathbb{R}_{+}^{n}\right\} \text {. }
$$

(iii) $\Gamma(I)$ is the union of all compact faces of $\Gamma_{+}(I)$.

(iv) $I=\left\langle g_{1}, \ldots, g_{s}\right\rangle$ is Newton non-degenerate if for each compact face $\triangle \subset \Gamma(I)$, the equations $g_{1 \triangle}(x)=g_{2 \triangle}(x)=\cdots=g_{s \triangle}(x)=0$ have no common solution in $(k-\{0\})^{n}$, where $g_{i}$ is the restriction of $g_{i}$ to the face $\triangle$, that is, if $g_{i}(x)=\sum a_{r} x^{r}$ then $g_{i \triangle}(x)=\sum_{r \in \triangle} a_{r} x^{r}$.

Definition 4.4. Let $h_{0}$ be $\mathcal{R}_{V}$-finitely determined and $J_{0}=$ $\left\langle d h_{0}\left(\alpha_{i}\right)\right\rangle_{i=1, \ldots, m}$. If $J_{0}$ is Newton non-degenerate we say that $h_{0}$ is Newton non-degenerate with respect to $V$.

We denote by $C\left(\overline{J_{0}}\right)$ the convex hull in $\mathbb{R}_{+}^{n}$ of the set $\left\{r:\left|x^{r}\right| \lesssim\right.$ $\left.\sup _{i}\left|d h_{0}\left(\alpha_{i}\right)\right|\right\}$. When $h_{0}$ is Newton non-degenerate with respect to $V$, it follows from Theorem 3.4 in [12] that $C\left(\overline{J_{0}}\right)=\Gamma_{+}\left(J_{0}\right)$. Taking the trivial filtration $w_{i}=1, i=1, \ldots, n$ in $k^{n}$ in the Theorem 4.1, then we get the following result:

THEOREM 4.5. Let $h_{0}$ be Newton non-degenerate with respect to $V$. Let $h(x, t)=h_{0}(x)+\operatorname{tg}(x)$ be a deformation of the germ $h_{0}$ with $\Gamma_{+}(g) \subset$ $\Gamma_{+}\left(J_{0}\right)$ and $\Gamma_{+}\left(d g\left(\alpha_{i}\right)\right) \subset \Gamma_{+}\left(J_{0}\right)$. Then $h$ is $C^{0}-\mathcal{R}_{V}$-trivial.

ExAmPle 4.6. Let $V, 0 \subseteq \mathbb{C}^{2}, 0$ be defined by $\varphi(x, y)=x^{3}-y^{2}=0$. The module $\Theta_{V}$ is generated by $\alpha_{1}=(2 x, 3 y)$ and $\alpha_{2}=\left(2 y, 3 x^{2}\right)$. In $[2$, Theorem 4.9], the $\mathcal{R}_{V}$ classification of germs $h: \mathbb{C}^{2}, 0 \rightarrow \mathbb{C}, 0$ is given, and we find the following normal form $h_{t}(x, y)=y^{2}+a x^{n}+t x^{n+1}, n \geq 4$, 
which is finitely determined for $a \neq 0$. Let $h_{0}(x, y)=y^{2}+a x^{n}$. Then $J_{0}=$ $\left\langle 2 a n x^{n}+6 y^{2}, 2 a n x^{n-1} y+6 x^{2} y\right\rangle$ is non-degenerate, hence $C\left(\overline{J_{0}}\right)=\Gamma_{+}\left(J_{0}\right)$. From Theorem 4.5, it follows that $h_{t}$ is $C^{0}-\mathcal{R}_{V}$-trivial.

EXAMPLE 4.7. Let $V, 0 \subseteq \mathbb{C}^{3}, 0$ be the swallowtail parameterized by $\left(x,-4 y^{3}-2 x y,-3 y^{4}-x y^{2}\right)$. The module $\Theta_{V}$ is generated by $\eta_{1}=$ $(2 x, 3 y, 4 z), \eta_{2}=\left(6 y,-2 x^{2}-8 z, x y\right)$ and $\eta_{3}=\left(-4 x^{2}-16 z,-8 x y, y^{2}\right)$. The $\mathcal{R}_{V}$ classification of germs $h: \mathbb{C}^{3}, 0 \rightarrow \mathbb{C}, 0$ given by Theorem 4.10 in [2], gives the normal form $h_{t}(x, y, z)=z+a x^{n}+t x^{n+1}, n \geq 2$ which is finitely determined for $a \neq 0, n \neq 2$, and $a \neq 0, a \neq 1 / 12, n=2$. Let $h_{0}(x, y, z)=$ $z+a x^{n}, J_{0}=\left\langle 2 a n x^{n}+4 z, 6 a n x^{n-1} y+x y,-4 a n x^{n+1}-16 a n x^{n-1} z+y^{2}\right\rangle$. From Theorem 4.5, $h_{t}$ is $C^{0}-\mathcal{R}_{V}$-trivial.

Acknowledgement. We are very grateful to the referee for suggesting the present formulation of Theorem 3.4 and for other useful comments. We would also like to thank Terence Gaffney and Marcelo Saia for helpful conversations.

\section{REFERENCES}

[1] J. W. Bruce and M. Roberts, Critical points of functions on analytic varieties, Topology, 27 (1988), no. 1, 57-90.

[2] J. W. Bruce, N. P. Kirk and A. A. du Plessis, Complete transversals and the classification of singularities, Nonlinearity, 10 (1997), 253-275.

[3] J. Damon, The unfolding and determinacy theorems for subgroups of $\mathcal{A}$ and $\mathcal{K}, \mathrm{Mem}-$ oirs Am. Math. Soc., 306 (1984).

[4] J. Damon, Topological triviality and versality for subgroups of $\mathcal{A}$ and $\mathcal{K}$, Memoirs Am. Math. Soc., 389 (1988).

[5] J. Damon, Deformations of sections of singularities and Gorenstein surface singularities, Am. Journal of Mathematics, 109 (1987), 695-722.

[6] J. Damon, Topological triviality and versality for subgroups of $\mathcal{A}$ and $\mathcal{K}$ : II. Sufficient conditions and applications, Nonlinearity, 5 (1992), 373-412.

[7] J. Damon, On the freeness of equisingular deformations of plane curve singularities, Topology and its Application, 118 (2002), 31-43.

[8] T. Fukui and L. Paunescu, Stratification theory from the weighted point of view, Canad. J. Math., 53 (2001), no. 1, 73-97.

[9] A. G. Kouchnirenko, Polyèdres de Newton et nombres de Milnor, Invent. Math., 32 (1976), 1-31.

[10] D. Mond, On the classification of germs of maps from $\mathbb{R}^{2}$ to $\mathbb{R}^{3}$, Proc. of London Math. Soc. 3, 50 (1985), 333-369.

[11] M. A. S. Ruas and M. J. Saia, $C^{l}$-determinacy of weighted homogeneous germs, Hokkaido Math. Journal, 26 (1997), 89-99. 
[12] M. Saia, The integral closure of ideals and the Newton filtration, J. Algebraic Geometry, 5 (1996), 1-11.

[13] J. N. Tomazella, Seções de Variedades Analíticas, Ph.D. Thesis, ICMSC-USP.

Maria Aparecida Soares Ruas

Instituto de Ciências Matemáticas e de Computação

Universidade de São Paulo, Departamento de Matemática

Caixa Postal 668

13560-970, São Carlos, SP

Brazil

maasruas@icmc .usp.br

João Nivaldo Tomazella

Departamento de Matemática

Universidade Federal de São Carlos

Caixa Postal 676

13560-905, São Carlos, SP

Brazil

tomazella@dm.ufscar.br 